\title{
ESTUDO PROSPECTIVO dO DERRAME PLEURAL PÓS-CIRURGIA ABDOMINAL E DOS FATORES DE RISCO ASSOCIADOS: AVALIAÇÃO POR ULTRA-SONOGRAFIA*
}

\author{
Luiz Antonio Rossi ${ }^{1}$, Sansom Henrique Bromberg ${ }^{2}$
}

Resumo MOTIVAC̣ÃO: Derrame pleural é alteração pulmonar comumente observada em exames de imagem após cirurgias abdominais eletivas, sem repercussão clínica na maioria dos enfermos, devendo ser individualizada das complicações pulmonares que requerem tratamento. Sua incidência, bem como os indicadores de risco, são desconhecidos em nosso meio. OBJETIVO: Determinar, pela ultra-sonografia, a incidência de derrame pleural pós-operatório (DPPO) em cirurgias abdominais eletivas e averiguar suas possíveis associações com fatores de risco relacionados aos doentes e procedimentos anestésico-cirúrgicos. MATERIAIS E MÉTODOS: Estudaramse $21(56,8 \%)$ mulheres e $16(43,2 \%)$ homens, entre 29 e 76 anos, submetidos a cirurgias abdominais eletivas. Os exames ecográficos foram realizados no pré-operatório e 48 horas após a cirurgia. Foram estudados os fatores de risco associados ao paciente - idade maior de 60 anos, sexo, obesidade, tabagismo, etilismo e presença de doenças associadas - , e ao procedimento anestésico-cirúrgico - cirurgia para ressecção de câncer, classe ASA $\geq 2$, tempo anestésico-cirúrgico, incisão longitudinal e incisão $>15 \mathrm{~cm}$. A litíase biliar $(43,2 \%)$ e a presença de câncer gastrintestinal $(43,2 \%)$ foram os principais responsáveis pela indicação cirúrgica. 0 DPPO foi graduado de pequeno, médio e grande. RESULTADOS: A incidência de DPPO foi de 70,3\% (26/37). Dois $(5,4 \%)$ desses doentes evoluíram com complicações pulmonares graves, um deles vindo a falecer. Idade maior de 60 anos, tabagismo, etilismo, obesidade e presença de doenças associadas não influenciaram o aparecimento de DPPO. Cirurgia para ressecção de câncer, classe ASA $\geq 2$, incisão longitudinal e incisão > 15 $\mathrm{cm}$ associaram-se de modo significante à presença de DPPO, que ocorreu mesmo na vigência de antibioticoprofilaxia. $O$ tempo de permanência hospitalar foi 2,4 vezes maior nos doentes com DPPO. CONCLUSÃO: A ocorrência de derrame pleural em pós-operatório de cirurgia abdominal eletiva é muito freqüente. A maioria dos DPPO é autolimitada, evoluindo de modo assintomático. A ecografia na constatação do DPPO mostrou-se efetiva e sua utilização merece ser difundida.

Unitermos: Abdome; Cirurgia; Complicações pós-operatórias; Complicação pulmonar; Fatores de risco; Ultrasonografia.

Abstract Pleural effusion following abdominal surgery and associated risk factors: ultrasound assessment. BACKGROUND: Pleural effusion is frequently seen on imaging examinations following elective abdominal surgery and has no clinical significance in most patients. This condition should be distinguished from pulmonary complications that require treatment. OBJECTIVE: To prospectively determine the incidence of pleural effusion in patients submitted to elective abdominal surgery using ultrasound (US), and to assess the possible association with risk factors related to the patients and anesthetic-surgical procedures. MATERIALS AND METHODS: Thirty-seven patients, $21(56.8 \%)$ female, and $16(43.2 \%)$ male aged 29 to 76 years submitted to elective abdominal surgery were evaluated. US was performed preoperatively and $\mathbf{4 8}$ hours after surgery in all patients. Associated risk factors were also assessed - age $>60$ years, sex, obesity, smoking history, alcoholism and associated diseases - , and anesthetic-surgical procedure - cancer resection, class ASA $\geq 2$, duration of surgery, longitudinal incision and incision $>15 \mathrm{~cm}$. Biliar lithiasis $(43.2 \%)$ and gastrointestinal cancer $(43.2 \%)$ were the main causes leading to surgery. RESULTS: The incidence of postoperative pleural effusion (PPE) detected by US was $70.3 \%$ (26/37). Two of these patients $(5.4 \%)$ developed pulmonary complications, and one died. The risk factors age $>60$ years, smoking history, alcoholism, obesity and associated diseases had no influence on the development of the PPE whereas cancer resection, class ASA $\geq 2$, longitudinal incision and incision $>15 \mathrm{~cm}$ were significantly statistically associated with the presence of PPE. PPE developed even during antibiotic therapy. The duration of hospitalization was more than 2.4 longer in the patients with PPE. CONCLUSION: PPE is a very frequent condition observed in patients submitted to elective abdominal surgery. Most of the cases of PPE are self-limited, resolving without symptoms. The use of the US for the detection of PPE proved to be effective and should therefore be recommended.

Key words: Abdomen; Surgery; Postoperative complications; Lung complications; Risk factors; Ultrasound.

* Trabalho realizado no Serviço de Radiologia e no Serviço de Gastroenterologia Cirúrgica do Hospital do Servidor Público Estadual "Francisco Morato de Oliveira” (HSPE-FMO), São Paulo, SP.

1. Professor Assistente da Disciplina Princípios de Radiolo- gia do Centro de Ciências Médicas e Biológicas da Pontifícia Universidade Católica de São Paulo (CCMB-PUC/SP).

2. Coordenador da Pós-Graduação em Gastroenterologia Cirúrgica do Instituto de Assistência Médica ao Servidor Público Estadual de São Paulo (IAMSPE).
Endereço correspondência: Prof. Dr. Luiz Antonio Rossi. Rua Cornélio Pires, 284, Condomínio Campos de Santo Antonio. Itu, SP, 13305-500. E-mail: luizrossimd@uol.com.br

Recebido para publicação em 24/6/2004. Aceito, após revisão, em 13/8/2004. 


\section{INTRODUÇÃO}

Mesmo com os avanços na técnica cirúrgica e dos procedimentos anestésicos, intensivos e quimioterápicos, a incidência de complicações pulmonares pós-operatórias (CPPO) permanece com índices elevados, principalmente após cirurgia do abdome superior ${ }^{(\mathbf{1})}$. Grandes são as oscilações desses índices, que variam de $1,5 \%$ até $87 \% \%^{(2-4)}$.

$\mathrm{O}$ termo complicações pulmonares tem sido utilizado para as diferentes alterações do órgão, quer para as sem repercussões clínicas - alterações pulmonares pósoperatórias (APPO) - propriamente ditas, quase sempre autolimitadas e assintomáticas, quer para aquelas com repercussões que agravam o estado clínico do enfermo ou retardam a alta hospitalar, as verdadeiras CPPO.

Quando se estabeleceram critérios objetivos definindo essas complicações, seus índices permaneceram constantes, ao redor de $20 \%$. Trabalho envolvendo 450 hospitais norte-americanos mostrou que a incidência de CPPO variou de $19 \%$ a $22 \%$ após cirurgia no andar superior do abdo$\mathrm{me}^{(2)}$. Entre nós, criterioso estudo constatou incidência de $14 \%{ }^{(5)}$.

Diferentes fatores de risco foram associados à presença de $\mathrm{CPPO}$, alguns relacionados ao paciente — idade, sexo, obesidade, tabagismo, etilismo e doenças associadas -, outros associados ao procedimento anestésico-cirúrgico - duração do ato operatório, porte e tipo da cirurgia, tamanho e tipo da incisão cirúrgica, permanência hospitalar, entre muitos outros.

Sabe-se, atualmente, que a função do sistema respiratório está invariavelmente comprometida durante e após a realização do ato cirúrgico ${ }^{(3)}$. Anestésicos e drogas utilizadas influenciam mecanismos centrais da respiração alterando o controle neural de importantes músculos, entre os quais o diafragma, dificultando o transporte mucociliar e liberando mediadores inflamatórios que produzem broncoconstrição ${ }^{(3,5,6)}$. Já o pneumoperitônio, a exsudação resultante do manuseio visceral e a distensão abdominal diminuem a complacência e alteram as propriedades mecânicas do compartimento abdominal, com maior prejuízo na função diafragmática ${ }^{(7)}$.
Poucos estudiosos preocuparam-se em observar a existência de derrames pleurais como possível alteração ou complicação pulmonar em doentes submetidos a cirurgias abdominais eletivas ${ }^{(4)}$.

$\mathrm{O}$ reconhecimento dos fatores de risco associados às APPO é de grande interesse para o cirurgião, já que estas, com freqüência, dão origem às $\mathrm{CPPO}^{(8)}$.

A incidência da alteração na imagem pleuropulmonar caracterizada como derrame pleural, bem como a dos indicadores de risco a ela associados são desconhecidas em nosso meio.

O objetivo deste estudo foi investigar, pela ultra-sonografia (US), a incidência de derrame pleural pós-operatório em doentes submetidos a cirurgias abdominais e avaliar suas possíveis associações com fatores de riscos relacionados ao doente e ao procedimento anestésico-cirúrgico.

\section{MATERIAIS E MÉTODOS}

Foram estudados, prospectivamente, 37 doentes adultos consecutivos, 21 (56,8\%) femininos e $16(43,2 \%)$ masculinos, com idade variando de 29 a 76 anos, média de $56,11 \pm 13,20$ anos, oriundos do Serviço de Gastroenterologia Cirúrgica do Hospital do Servidor Público Estadual "Francisco Morato de Oliveira". O estudo foi analisado e aprovado pela Comissão de Ética e Pesquisa deste hospital. Os doentes, devidamente cientificados, consentiram em atender o protocolo nas suas diferentes etapas. Todos foram submetidos a diferentes cirurgias abdominais durante os anos de 1999 e 2000.

Os doentes foram agrupados em duas faixas etárias: menos de 60 anos e igual ou maior que 60 anos. A altura dos pacientes variou de 150 a $180 \mathrm{~cm}$ (média de $164 \pm$ $8,56 \mathrm{~cm}$ ). O peso variou de 40 a $110 \mathrm{~kg}$ (média de 70,89 $\pm 14,69 \mathrm{~kg}$ ). O índice de massa corporal (IMC), calculado segundo critérios da Organização Mundial da Saúde (OMS) (relação entre o peso em quilogramas e o quadrado da altura em centímetros) considerou obeso o paciente com índice igual ou superior a 30. Foram considerados tabagistas crônicos doentes com consumo de mais de 20 maços/ano e etilistas crônicos os que ingeriam mais de $80 \mathrm{~g}$ de álcool por dia.
A litíase biliar (43,2\%) e a presença de câncer digestivo $(43,2 \%)$ foram os principais responsáveis pela indicação cirúrgica. Os procedimentos cirúrgicos realizados podem ser vistos na Tabela 1 .

Tabela 1 Cirurgias abdominais praticadas em 37 doentes.

\begin{tabular}{|l|r|r|}
\hline Cirurgias & N & \multicolumn{1}{c|}{$\%$} \\
\hline Colecistectomia com ERVB $^{*}$ & 17 & 46,0 \\
Colectomia esquerda $^{\dagger}$ & 7 & 18,9 \\
Gastrectomia ampliada $^{\dagger}$ & 6 & 16,2 \\
Gastroduodenopancreatectomia $^{\dagger}$ & & \\
parcial $^{\dagger}$ & 2 & 5,4 \\
Exérese de delgado $^{\text {Colectomia direita }^{\dagger}}$ & 2 & 5,4 \\
Drenagem de abscesso hepático & 1 & 2,7 \\
Exploração do retroperitônio $^{\dagger}$ & 1 & 2,7 \\
\hline Total $^{*}$ & 37 & 100,7 \\
\hline
\end{tabular}

* ERVB, exploração radiológica das vias biliares; ${ }^{\dagger}$ Pacientes com câncer digestivo.

A hipertensão arterial foi a doença associada predominante, tendo ocorrido em $21,6 \%(8 / 37)$ dos doentes, seguida pela bronquite crônica, que incidiu em 10,8\% (4/37) dos enfermos. Diabetes mellitus e hepatopatias crônicas estavam presentes em 5,4\% (2/37) deles.

O mesmo procedimento anestésico foi utilizado em todos os doentes: anestesia geral inalatória e endovenosa. Em dez pacientes a anestesia peridural ou raquidiana foi associada à anestesia geral. Todos os doentes foram classificados pela proposição da American Society of Anesthesiologists (ASA).

Foram devidamente registrados os seguintes eventos: doentes que receberam antibioticoprofilaxia, o tempo de duração do ato cirúrgico, o tipo e o tamanho da incisão utilizada nos procedimentos cirúrgicos (exceto em dois pacientes submetidos a cirurgia videolaparoscópica), o tempo de permanência hospitalar em dias após a cirurgia e a ocorrência de complicações pós-operatórias.

Os principais fatores de risco estudados, bem como suas distribuições, podem ser observados na Tabela 2.

Os exames de US foram realizados com aparelho Aloka 2000, transdutor multifreqüencial de $5 \mathrm{MHz}$. Os enfermos foram examinados sentados, com o transdutor 
Tabela 2 Fatores de risco de 37 doentes e sua distribuição.

\begin{tabular}{|l|l|r|c|}
\hline Fator de risco & Categoria & $\mathrm{N}$ & $\%$ \\
\hline Idade & $<60$ & 18 & 48,6 \\
IMC & $\geq 60$ & 19 & 51,4 \\
Tabagismo & $<30$ & 31 & 83,8 \\
& $\geq 30$ & 6 & 16,2 \\
Etilismo & Ausente & 24 & 64,9 \\
& Presente & 13 & 35,1 \\
Doenças associadas & Ausente & 32 & 86,4 \\
& Presente & 5 & 13,6 \\
Câncer & Ausente & 26 & 70,3 \\
& Presente & 11 & 29,7 \\
ASA & Ausente & 20 & 54,1 \\
& Presente & 17 & 45,9 \\
Incisão & $<2$ & 17 & 45,9 \\
& $\geq 2$ & 20 & 54,1 \\
Incisão & Transversa & 17 & 48,6 \\
& Longitudinal & 18 & 51,4 \\
Duração da cirurgia & $\leq 15 \mathrm{~cm}$ & 18 & 51,4 \\
& $>15 \mathrm{~cm}$ & 17 & 48,6 \\
& $<210 \mathrm{~min}$ & 22 & 59,5 \\
& $\geq 210 \mathrm{~min}$ & 15 & 40,5 \\
\hline
\end{tabular}

orientado entre os espaços intercostais para obtenção da janela acústica. Em cinco deles o estudo ecográfico foi realizado na posição de decúbito lateral, devido ao quadro doloroso pós-operatório. O exame foi considerado anormal quando da constatação de coleção líquida livre no espaço pleural - derrame pleural —, que se manifestou como área hipoecogênica com reforço acústico posterior. O relatório foi fornecido por um só observador, sendo classificado, segundo critério subjetivo, em pequeno $(+)$, moderado $(++)$ ou grande $(+++)$, como ilustra a Figura 1.

Os dados foram analisados utilizando pacote de "software" SPSS 6.0 (SPSS, Chicago) e SAS for Windows (SAS, Cary, NC). Sumário descritivo estatístico foi computado para todas as variáveis, incluindo freqüência, proporções, médias e desvios-padrão. Correlações para fatores de risco e derrame pleural foram analisadas usando testes do qui-quadrado e exato de Fisher. Para comparação da presença/ausência de derrame pleural em relação ao tempo de permanência hospitalar, foi realizado o teste de Mann-Whitney. Todos os testes foram realizados admitindo-se nível de significância de $5 \%(p=0,05)$. Os valores significantes receberam asterisco $(*)$ e os não significantes foram assinalados pela sigla NS.

\section{RESULTADOS}

A incidência de derrame pleural constatado pela US foi de 70,3\% (26/37). O derrame pleural foi de pequeno volume em $53,8 \%$ (14/37) dos doentes, moderado em $19,3 \%(5 / 37)$ e grande em $26,9 \%(7 / 37)$. A incidência de derrame pleural por tipo de cirurgia, em ordem decrescente, foi:
Figura 1. A: US de tórax normal, mostrando os compartimentos torácico e abdominal separados por linha hiperecogênica que representa o diafragma (estrelas), sem líquido no espaço pleural. B,C,D: US mostrando coleções líquidas no espaço pleural de pequeno, médio e grande volume, respectivamente (setas), bem delimitados pelo diafragma (estrela) e presença de reforço acústico (R), nos dois últimos.

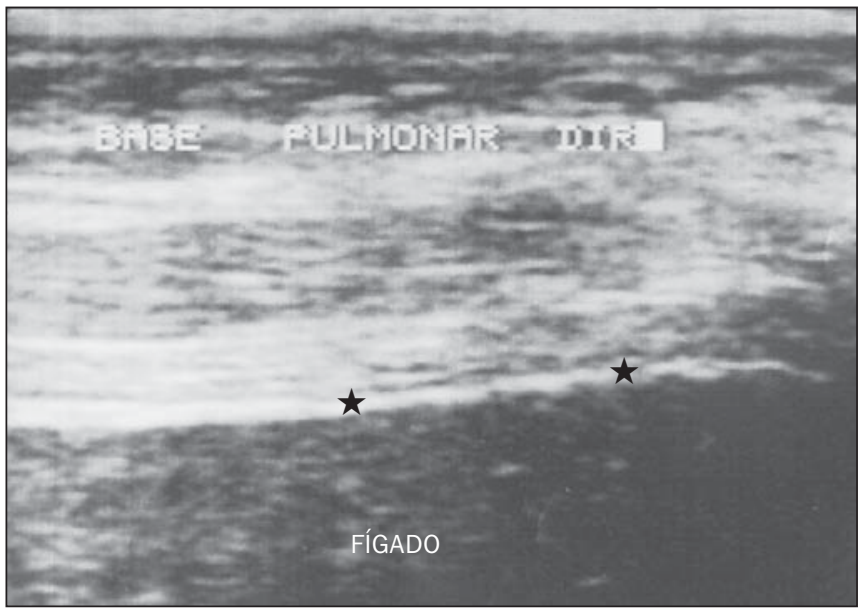

A

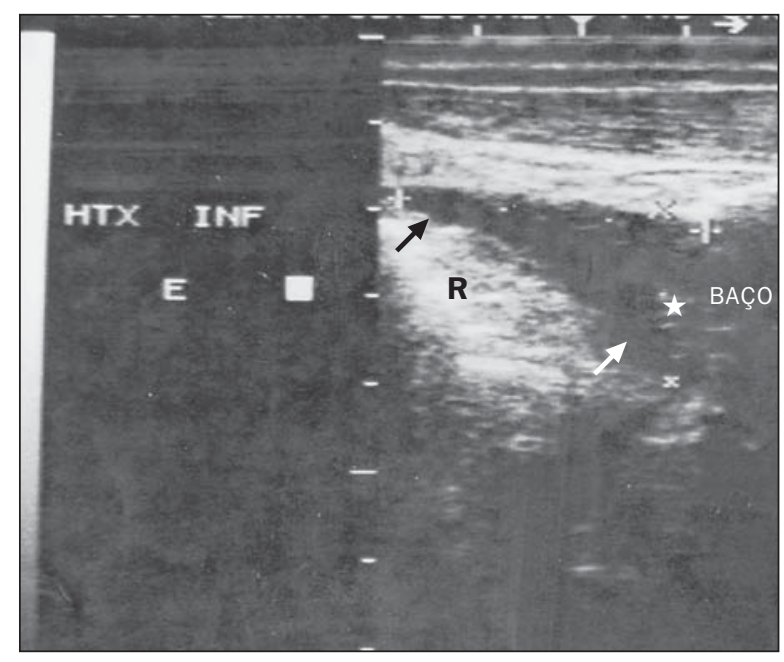

c

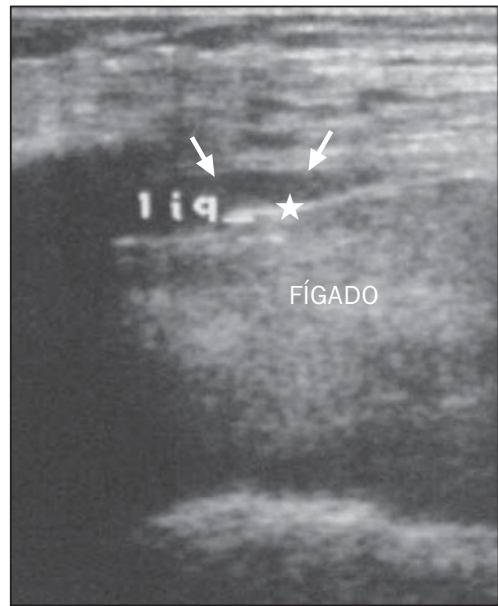

B

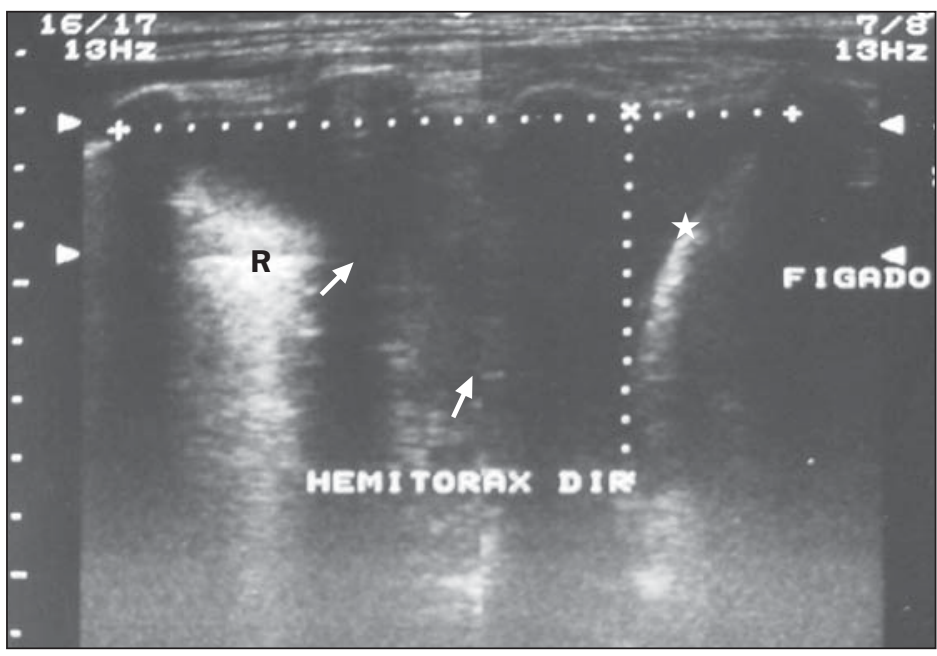

D 
colo-retal, 100,0\% (8/8); ressecção de delgado, 100,0\% (2/2); exploração do retroperitônio, 100,0\% (1/1); gastroduodenal, 71,4\% (5/7); hepatobiliar, 52,6\% (10/19). Dos 26 doentes com derrame pleural, dois $(7,7 \%)$ evoluíram com pneumonia grave; um deles veio a falecer e o outro se curou.

A média de tempo de permanência hospitalar foi significantemente maior nos doentes que desenvolveram derrame pleural quando comparados aos sem derrame - 5,89 dias $\times 2,86$ dias - (Tabela 3).

A idade, a obesidade, o tabagismo, o etilismo, a presença de doenças associadas e a duração do procedimento cirúrgico não se associaram à presença de derrame pleural. O derrame pleural se associou de modo significante aos fatores de risco enumerados na Tabela 4.

Tabela 3 Relação entre derrame pleural detectado por US e dias de internação após a cirurgia.

\begin{tabular}{|l|l|l|l|}
\hline Variável & Derrame pleural & N & Média \\
\hline $\begin{array}{l}\text { Permanência } \\
\text { hospitalar (dias) }\end{array}$ & Ausência & 7 & 2,86 \\
& Presença & 29 & 6,89 \\
\hline
\end{tabular}

Teste Mann-Whitney; $p=0,0124 *$.

Tabela 4 Fatores de risco associados a derrame pleural.

\begin{tabular}{|l|c|}
\hline Fator de risco & $\begin{array}{c}\text { Derrame pleural } \\
(p)\end{array}$ \\
\hline Câncer & $0,003^{*}$ \\
ASA $\geq 2$ & $0,004^{\dagger}$ \\
Incisão longitudinal & $<0,001^{\star}$ \\
Incisão $>15 \mathrm{~cm}$ & $0,018^{*}$ \\
\hline
\end{tabular}

* Teste exato de Fisher. ${ }^{\dagger}$ Teste qui-quadrado.

\section{DISCUSSÃO}

Alterações pulmonares constatadas em exames radiológicos são seqüelas comuns no pós-operatório de cirurgias abdominais, caracterizando-se pela ausência de repercussões clínicas na grande maioria dos enfermos. Devem, por isso, ser diferenciadas das complicações pulmonares, que ocorrem em proporções menos significativas.

Vários agentes estariam implicados no desenvolvimento do derrame pleural. A irritação local diafragmática seria um dos principais, podendo se dar por manuseio, por fluidos intracavitários de natureza química ou infecciosa e por lesões hemorrágicas despercebidas ${ }^{(\mathbf{9})}$. A ruptura traumá- tica das conexões linfáticas por ocasião de descolamentos subdiafragmáticos ou da presença de efusão ou líquido peritoneal seria outro fator de importância ${ }^{(\mathbf{1 0 , 1 1})}$. A imobilidade pós-operatória, o desequilíbrio hidroeletrolítico e a concomitância com ascite também são lembrados ${ }^{(\mathbf{1 2})}$.

$\mathrm{O}$ alto índice de derrame pleural pósoperatório verificado nos exames ecográficos deste trabalho - 70,3\% (26/37) atestou a sensibilidade deste exame.

Dois $(7,7 \%)$ dos enfermos apresentaram broncopneumonia grave enquanto estavam internados, vindo um a falecer por esse motivo, confirmando a gravidade da broncopneumonia pós-operatória ${ }^{(1,13,14)}$.

Constata-se, com o aumentar da idade, decréscimo na força muscular inspiratória e expiratória, prejudicando a capacidade de inspiração profunda, o mecanismo de tosse e a capacidade de clarear secreções, principalmente na posição supina ${ }^{(3)}$. Muitos estudiosos verificaram índices expressivos de CPPO em enfermos com idade acima de $50 \operatorname{anos}^{(\mathbf{5 , 8 , 1 3})}$. Exame de 153 doentes isentos de doenças associadas e sem outros indicadores de risco, submetidos a cirurgia do andar superior do abdome, mostrou significante incidência de CPPO $(51,0 \%)$ naqueles com idade entre 50 e 70 anos comparados aos com menos de 50 anos $(30,0 \%)^{(\mathbf{1 5})}$. Estudo recente avaliando 400 doentes submetidos a cirurgia abdominal mostrou incidência de CPPO significantemente maior na população com mais de 60 anos de idade $32,0 \% \times 17,0 \%{ }^{(16)}$.

Na presente pesquisa não se notou associação de derrame pleural com a idade. Embora a maior parte dos enfermos com derrame pleural tivesse mais de 60 anos $79,0 \% \times 61,1 \%-$, esses valores não foram significantes. $\mathrm{O}$ melhor preparo dos idosos para cirurgias eletivas parece justificar, pelo menos parcialmente, esses resultados. A essa mesma conclusão chegaram outros estudiosos $^{(\mathbf{1 7 - 2 0})}$.

$\mathrm{A}$ associação da obesidade às $\mathrm{CPPO}$ ainda é motivo de controvérsias. Alguns autores sugeriram ser a obesidade importante fator de risco associado às CPPO, embora não constatassem significância estatística para tal fato ${ }^{(\mathbf{2 1 - 2 4})}$. Somente dois trabalhos constataram influência da obesidade no desenvolvimento das CPPO, embora tenham utilizado doentes pré-obesos em suas amostras ${ }^{(\mathbf{1 3}, \mathbf{1 6})}$. Portadores de obesidade mórbida submetidos a cirurgia de redução gástrica apresentaram baixo índice de CPPO $(3,9 \%)^{(\mathbf{2 4})}$. Recente artigo de revisão não credenciou a obesidade como fator de risco para gênese destas $\mathrm{CPPO}^{(\mathbf{2 5})}$.

Este estudo não associou a presença de obesidade a maior risco de derrame pleural. O cuidadoso preparo pré-operatório destes doentes, a inexistência de cirurgias emergenciais, a atenção durante todo o ato anestésico-cirúrgico e pós-operatório imediato pelas diferentes equipes seguramente contribuíram para os resultados obtidos. Os dados contraditórios no estudo deste indicador de risco se devem, entre outras razões, ao pequeno número de trabalhos prospectivos, à falha na uniforme definição de obesidade e à discordância na definição de alterações e complicações pulmonares.

A íntima relação entre o hábito de fumar e bronquite crônica, levando ao aumento progressivo da secreção e constrição da árvore brônquica, predispõe associar o tabagismo como fator de risco de importância no aparecimento de CPPO. Entretanto, muitos aspectos ligados a esta variável ainda estão por serem mais bem esclarecidos. O consumo de fumo nas últimas oito semanas que precedem o ato cirúrgico e o de 20 maços/ano de cigarros ou mais desempenhariam importante papel no aparecimento de $\mathrm{CPPO}^{(\mathbf{6 , 1 6 , 2 1 )}}$. O tabagismo associou-se a incidência significante de

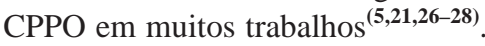

Constatou-se, no presente estudo, incidência ligeiramente superior de derrame pleural nos enfermos fumantes - 84,1\% $\times 62,5 \%$-, sem alcançar significância estatística. De modo semelhante, outros trabalhos não associaram a presença de tabagismo com disfunções pulmonares ${ }^{(\mathbf{4}, \mathbf{1 3}, \mathbf{1 5})}$.

$\mathrm{O}$ etilismo não se associou à presença de derrame pleural neste estudo, embora se constatasse incidência maior no grupo com derrame pleural. Dentre os poucos estudos a respeito também foi constatada maior incidência de CPPO em doentes com história de consumo alcoólico - 65,0\% $\times$ $52,0 \%$ - , porém sem significância estatística $^{(26)}$. A frequiente associação do etilismo com doenças hepatopancreáticas com níveis séricos rebaixados de albumina poderia levar esses doentes, quando submetidos 
a cirurgia abdominal, a apresentar com maior freqüência APPO ou CPPO.

A freqüência da doença pulmonar obstrutiva crônica (DPOC) no pré-operatório de doentes com afecções abdominais é alta e contribui de modo expressivo na eleva-

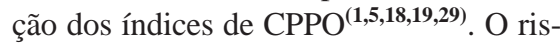
co relativo do aparecimento dessas complicações em doentes com DPOC aumentou de 2,7 a 4,7 vezes $^{(\mathbf{3 0})}$. Outros estudiosos não encontraram associação entre DPOC e o aparecimento de $\mathrm{CPPO}^{(\mathbf{8 , 1 6})}$. Os motivos desses resultados contraditórios repousaram no número desigual e extremamente discordante dos doentes estudados, o método utilizado no reconhecimento da DPOC e a falta de uniformização de critérios na definição das CPPO.

Moléstias associadas como hipertensão arterial ativa, cardiopatias e diabetes mellitus em enfermos submetidos a cirurgias abdominais prolongadas aumentaram a probabilidade de CPPO em 47,0\% ${ }^{(\mathbf{5})}$.

Apesar da maior incidência de derrame pleural nos doentes com moléstias associadas deste estudo - 91,9\% × 61,5\% —, os valores não foram significantes.

Escassos registros da literatura mostraram maior incidência de CPPO em portadores de doenças malignas submetidos a tratamento cirúrgico ${ }^{(\mathbf{1 9 , 2 8})}$. A deficiência nutricional de alguns desses doentes contribui no desenvolvimento dessas complicações, por deprimir as funções respiratórias por queda da IgA secretora, diminuição da resposta ventilatória à hipóxia, além de interferir na dinâmica respiratória determinando hipotrofia muscular ${ }^{(31)}$. Os enfermos com câncer deste estudo apresentaram taxa significantemente maior de derrame pleural $-94,1 \% \times 50 \%$. As razões que justificariam a associação deste indicador de risco com a presença de derrame pleural estariam ligadas a linfadenectomia e descolamentos mais extensos, à secção de maior número de linfáticos e ao maior tempo anestésico-cirúrgico.

A classificação ASA previu o aparecimento de CPPO em vários estudos ${ }^{(6,13,20,32)}$. Alguns autores julgaram-na o mais importante indicador de risco dessas complicações, já que $88,0 \%$ dos seus enfermos da classe ASA > 1 vieram a apresentá-las ${ }^{(13)}$.

A incidência de derrame pleural neste estudo foi significantemente maior nos doentes classificados como ASA $\geq 2$ $90 \% \times 47,1 \%$. Fator importante na adoção dessa variável como indicador de risco é o fato de poder ser estimada com facilidade no pré-operatório. As críticas a ela endereçadas consistem em ser operadordependente e a necessidade de confiar na avaliação subjetiva do examinador ${ }^{(23,27)}$.

Cirurgiões que adotam a incisão transversa justificam sua preferência por ela proporcionar bom acesso cirúrgico, menos dor em pós-operatório imediato, melhor resultado estético em longo prazo e menores índices de hérnia incisional ${ }^{(\mathbf{3 3})}$.

Na população deste estudo constatouse derrame pleural em 94,5\% (17/18) daqueles submetidos à incisão longitudinal contra 53,0\% (9/17) dos submetidos à incisão transversa, e esses valores mostraramse significantes.

Diversos estudos mostraram associação significante entre incisão cirúrgica longitudinal e a presença de $\mathrm{CPPO}^{(\mathbf{1 6}, 19)}$. Estudo comparativo da incisão subcostal com a mediana no tratamento cirúrgico da litíase biliar de 129 doentes mostrou menor prejuízo da função pulmonar em termos de capacidade vital e volume expiratório forçado em um segundo nos submetidos a incisões subcostais, menos dor pós-operatória e menor tempo de permanência hospitalar $^{(\mathbf{1 8})}$. A menor intensidade da dor pósoperatória apresentada por doentes submetidos a incisões transversas possibilita melhor desempenho da musculatura do abdome superior no ato respiratório, o que resultaria em melhor função pulmonar e menor índice de complicações ${ }^{(34)}$.

Os enfermos do presente estudo submetidos a incisões maiores de $15 \mathrm{~cm}$ tiveram incidência significantemente maior de derrame pleural quando comparados àqueles com incisões menores - 94,2\% $\times 55,6 \%$.

Em doentes submetidos a colecistectomia a função pulmonar pós-operatória foi significantemente maior nos doentes com pequenas incisões quando comparadas com incisões maiores ${ }^{(35)}$.

A ruptura de fibras dos músculos auxiliares dos movimentos respiratórios e o quadro doloroso provocado pelas incisões abdominais seriam responsáveis pela diminuição da atividade respiratória, o que justificaria o maior dano causado por grandes incisões ${ }^{(\boldsymbol{6})}$. Também por essas razões, a ci- rurgia abdominal laparoscópica propiciaria menor prejuízo na função pulmonar e melhor saturação de oxigênio pós-operatória do que a cirurgia aberta ${ }^{(36)}$.

Não se constatou influência da duração do procedimento anestésico-cirúrgico nos índices de derrame pleural, embora estas taxas tenham sido maiores nos procedimentos de longa duração $-80 \% \times$ $53,8 \%$. Outros estudiosos também não constataram associação entre maior tempo despendido no ato cirúrgico e aumento das $\mathrm{CPPO}^{(15)}$. No entanto, diferentes estudos constataram que procedimento cirúrgico de longa duração - mais de três horas associou-se a risco aumentado de CPPO, provavelmente pela maior manipulação visceral $^{(5,16,19,27,36)}$. Por sua vez, em 148 doentes estudados por Mitchell et al., verificou-se que a duração do tempo anestésico foi significantemente maior em portadores de CPPO (114,5 minutos) quando comparado aos que não apresentaram complicações $(102,6 \text { minutos })^{(\mathbf{2 0})}$. Estudo brasileiro mostrou que no grupo de 36 $(12,1 \%)$ doentes com CPPO, 89,0\% foram submetidos a cirurgia de longa duração ${ }^{(21)}$. Esse indicador de risco foi considerado entre os mais importantes nos associados ao aparecimento das CPPO.

Na pesquisa da literatura, trabalhos utilizando análise multivariada identificaram como principais fatores de risco para CPPO: a idade acima de 60 anos, obesidade, tabagismo, classe ASA $\geq 2$, cirurgia de neoplasias malignas, cirurgia de longa duração, incisões longitudinais e de grande extensão ${ }^{(\mathbf{5}, 13,16,20,28)}$.

Fundamentalmente, este estudo comprovou ser o derrame pleural sequiela comum de operações abdominais. O emprego da US na constatação do derrame pleural pós-operatório mostrou-se efetivo e sua utilização merece ser difundida. As alterações constatadas evoluíram para complicações em número pequeno de doentes, mas foram de natureza grave. Se raramente aparecem como evento de importância clínica, sendo usualmente autolimitada, elas foram capazes de prolongar a permanência hospitalar dos enfermos.

Futuros ensaios, em populações cirúrgicas mais numerosas e homogêneas, poderão melhor sedimentar o conhecimento sobre o tema. 


\section{REFERÊNCIAS}

1. Wightman JA. A prospective survey of the incidence of postoperative pulmonary complications. Br J Surg 1968;55:87-91.

2. O'Donohue WJ Jr. Postoperative pulmonary complications. When are preventive and therapeutic measures necessary? Postgrad Med 1992;91:16775.

3. Celli BR. What is the value of preoperative pulmonary function testing? Med Clin North Am 1993; 77:309-25.

4. Torrington KG, Bilello JF, Hopkins TK, Hall EA Jr. Postoperative pulmonary changes after laparoscopic cholecystectomy. South Med J 1996;89: 675-8

5. Pereira ED, Fernandes AL, Anção MS, Peres CA, Atallah NA, Faresin SM. Prospective assessment of the risk of postoperative pulmonary complications in patients submitted to upper abdominal surgery. São Paulo Med J 1999;117:151-60.

6. Warner DO. Preventing postoperative pulmonary complications: the role of the anesthesiologist Anesthesiology 2000;92:1467-72.

7. Dureuil B, Cantineau JP, Desmonts JM. Effects of upper or lower abdominal surgery on diaphragmatic function. Br J Anaesth 1987;59:1230-5.

8. Fujita T, Sakurai K. Multivariate analysis of risk factors for postoperative pneumonia. Am J Surg 1995;169:304-7.

9. Light RW. Doenças da pleura. Rio de Janeiro: Revinter, 2001.

10. Ekman H, Odman P. The occurrence of pleural effusion following biliary surgery; a clinical-roentgenologic study. Acta Chir Scand 1951;101:41928.

11. Lieberman FL, Hidemura R, Peters RL, Reynolds TB. Pathogenesis and treatment of hydrothorax complicating cirrhosis with ascites. Ann Intern Med 1966;64:341-51.

12. Shipley RT. The chest radiograph after extrathoracic surgery. Semin Roentgenol 1988;23:49-60.
13. Hall JC, Tarala RA, Hall JL, Mander J. A multivariate analysis of the risk of pulmonary complications after laparotomy. Chest 1991;99:923-7.

14. Mathew JT, D'Souza GA, Kilpadi AB. Respiratory complications in postoperative patients. J Assoc Physicians India 1999;47:1086-8.

15. Roukema JA, Carol EJ, Prins JG. The prevention of pulmonary complications after upper abdominal surgery in patients with noncompromised pulmonary status. Arch Surg 1988;123:30-4.

16. Brooks-Brunn JA. Predictors of postoperative pulmonary complications following abdominal surgery. Chest 1997;111:564-71.

17. Mitchell C, Garrahy P, Peake P. Postoperative respiratory morbidity: identification and risk factors. Aust N Z J Surg 1982;52:203-9.

18. Garcia-Valdecasas JC, Almenara R, Cabrer C, et al. Subcostal incision versus midline laparotomy in gallstone surgery: a prospective and randomized trial. Br J Surg 1988;75:473-5.

19. Dilworth JP, White RJ. Postoperative chest infection after upper abdominal surgery: an important problem for smokers. Respir Med 1992;86:20510 .

20. Mitchell CK, Smoger SH, Pfeifer MP, et al. Multivariate analysis of factors associated with postoperative pulmonary complications following general elective surgery. Arch Surg 1998;133:194-8.

21. Saad IA, Zambon L. Variáveis clínicas de risco préoperatório. Rev Assoc Med Bras 2001;47:117-24.

22. Pemberton LB, Manax WG. Relationship of obesity to postoperative complications after cholecystectomy. Am J Surg 1971;121:87-90.

23. Luce JM. Respiratory complications of obesity. Chest 1980;78:626-31.

24. Pasulka PS, Bistrian BR, Benotti PN, Blackburn GL. The risks of surgery in obese patients. Ann Intern Med 1986;104:540-6.

25. Williams-Russo P, Charlson ME, MacKenzie CR, Gold JP, Shires GT. Predicting postoperative pulmonary complications. Is it a real problem? Arch Intern Med 1992;152:1209-13.
26. Ephgrave KS, Kleiman-Wexler R, Pfaller M, Booth B, Werkmeister L, Young S. Postoperative pneumonia: a prospective study of risk factors and morbidity. Surgery 1993;114:815-21.

27. Lawrence VA, Dhanda R, Hilsenbeck SG, Page CP. Risk of pulmonary complications after elective abdominal surgery. Chest 1996;110:744-50.

28. Fuso L, Cisternino L, Di Napoli A, et al. Role of spirometric and arterial gas data in predicting pulmonary complications after abdominal surgery. Respir Med 2000;94:1171-6.

29. Pereira ED, Faresin SM, Fernandes AL. Morbidade respiratória nos pacientes com e sem síndrome pulmonar obstrutiva submetidos a cirurgia abdominal alta. Rev Assoc Med Bras 2000;46:15-22.

30. Smetana GW. Preoperative pulmonary evaluation. N Engl J Med 1999;340:937-44.

31. Baier H, Somani P. Ventilatory drive in normal man during semistarvation. Chest 1984;85:222-5.

32. Wong DH, Weber EC, Schell MJ, Wong AB, Anderson CT, Barker SJ. Factors associated with postoperative pulmonary complications in patients with severe chronic obstructive pulmonary disease. Anesth Analg 1995;80:276-84.

33. Bromberg SH, Waisberg J, Gonçalves JE, Zanoto A, Godoy AC, Goffi FS. Tratamento cirúrgico da litíase biliar em idosos: experiência em hospital de ensino. Rev Col Bras Cir 1998;25:161-6.

34. Becquemin JP, Piquet J, Becquemin MH, Melliere D, Harf A. Pulmonary function after transverse or midline incision in patients with obstructive pulmonary disease. Intensive Care Med 1985;11:247-51

35. Lindell P, Hedenstierna G. Ventilation efficiency after different incisions for cholecystectomy. Acta Chir Scand 1976;142:561-5.

36. McMahon AJ, Russell IT, Ramsay G, et al. Laparoscopic and minilaparotomy cholecystectomy: a randomized trial comparing postoperative pain and pul-monary function. Surgery 1994;115:533-9. 